\title{
LIGAÇÃO ENTRE PESQUISA E EXTENSÃO NA MANIPULAÇÃO DE ALIMENTOS EM UMA COOPERATIVA AGROINDUSTRIAL
}

\author{
Luana Késia dos Santos Fialho ${ }^{1}$ \\ ORCID: 0000-0002-0077-9822 \\ Pedro Paullo Alves dos Santo ${ }^{1}$ \\ ORCID: 0000-0002-2480-0665 \\ Gabrieli da Silvia Ferrari ${ }^{1}$ \\ ORCID: 0000-0001-8199-1392 \\ Maira Helena Carvalho Pereira ${ }^{1}$ \\ ORCID: 0000-0003-3622-3858 \\ MARIANA MANFROI FUZINATTO²
ORCID: 0000-0002-6678-6654
}

Resumo: Esse projeto de extensão foi desenvolvido no município de Naviraí-MS, objetivando a melhoria da qualidade dos alimentos produzidos e comercializados por uma cooperativa agroindustrial do município, visando o bem estar e a saúde dos consumidores. O objetivo de aplicar as noções básicas para os manipuladores de alimentos da forma correta de aplicação, monitoramento e elaboração de procedimentos operacionais padronizados (POP) e Boas Práticas de Manipulação (BPM), através de palestras, treinamentos e elaboração de um material informativo com fundamento na Resolução RDC N 216 de 15 de setembro de 2004 para incentivar a adoção de ações corretivas. A experiência obtida bem como dos manipuladores de alimentos da cooperativa participantes mostraram a importância do Projeto de Extensão voltados para esse público, possibilitando uma imersão de estudos dos alunos para além da

1 Discentes do curso de Engenharia de Alimentos, Universidade Estadual de Mato Grosso do Sul (UEMS), Naviraí, Mato Grosso do Sul, Brasil.

2 Docente do curso de Engenharia de Alimentos, Universidade Estadual de Mato Grosso do Sul (UEMS), Naviraí, Mato Grosso do Sul, Brasil. 
sala de aula, além de contribuir com a melhoria da qualidade dos alimentos comercializados no município. .

Palavras-chave: Relação pesquisa-extensão. Boas práticas. Treinamentos. Higiene pessoal.

\title{
CONNECTION BETWEEN RESEARCH AND EXTENSION IN FOOD MANIPULATION IN AN AGRO-INDUSTRIAL COOPERATIVE
}

\begin{abstract}
This extension project was developed in the municipality of Naviraí-MS, aiming at improving the quality of food produced and marketed by an agroindustrial cooperative of the municipality, aiming at the well-being and health of consumers. The objective of applying the basics for food handlers to the correct application, monitoring and elaboration of standard operating procedures (POPs) and Good Handling Practices (BPM), through lectures, training and elaboration of informational material based in Resolution RDC No. 216 of September 15, 2004 to encourage the adoption of corrective actions. The experience gained as well as the food handlers of the participating cooperative showed the importance of the Extension Project aimed at this audience, allowing students to immerse themselves in studies beyond the classroom, as well as contribute to the improvement of the quality of the foods commercialized in the County.
\end{abstract}

Keywords: Research-extension relationship, good practices, training, personal hygiene.

\section{CONEXIÓN ENTRE INVESTIGACIÓN Y EXTENSIÓN EN LA MANIPULACIÓN DE ALIMENTOS EN UNA COOPERATIVA AGROINDUSTRIAL}

Resumen: Este proyecto de extensión fue desarrollado en el municipio de Naviraí-MS, con el objetivo de mejorar la calidad de los alimentos producidos y comercializados por una cooperativa agroindustrial del municipio, buscando el bienestar y la salud de los consumidores. El objetivo de aplicar las nociones básicas para los manipuladores de alimentos de la forma correcta de aplicación, monitoreo y elaboración de procedimientos operacionales estandarizados (POP) y Buenas Prácticas de Manipulación (BPM), a través de conferencias, entrenamientos y elaboración de un material informativo con fundamento en la Resolución RDC N ${ }^{\circ} 216$ de 15 de septiembre de 2004 para incentivar la adopción de acciones correctivas. La experiencia obtenida así como de los manipuladores de alimentos de la cooperativa participantes mostraron la importancia del Proyecto de Extensión orientados a ese público, posibilitando una inmersión de estudios de los alumnos más allá del aula, además de contribuir con la mejora de la calidad de los alimentos comercializados en el mercado municipio.

Palabras clave: Relación investigación-extensión, buenas prácticas, entrenamientos, higiene personal. 


\section{INTRODUÇÃO}

As cooperativas agroindustriais têm como finalidade etapas de processamentos e transformação da matéria prima, tendo com princípio a comercialização dos mesmos (FLORES et al., 2010). Os procedimentos de Boas Práticas de Manipulação (BPM) abordam medidas a serem tomadas por indústrias alimentícias e pequenas propriedades, garantindo um alimento seguro para o consumo. Deste modo, existem legislações que regulamentam medidas técnicas em determinada linha de produção. (MACHADO et al. 2015).

Os conceitos teóricos cooperativos são: igualdade; democracia; solidariedade e fraternidade. As ações cooperativas dominam no agronegócio como modelos competentes de coordenação diminuindo os perigos para o fabricante frente ao mercado e acrescentando valor (BIALOSKORSKI, 2000).

De acordo com Rio (2004), os diferentes setores alimentícios estão cada vez mais exigentes com a segurança e qualidade dos alimentos oferecidos aos consumidores.

Ultimamente as indústrias do Brasil estão mais preocupadas em relação de garantir que os produtos que produzem tenha de uma forma efetiva as perspectivas dos seus consumidores voltados aos aspecto referente a qualidade dos alimentos. A elaboração do código de defesa do consumidor juntamente a atuação de órgãos como por exemplo a coordenadoria de proteção e defesa do consumidor - PROCON, sendo assim, contribuíram de modo essencial para as preocupação das indústrias; essas instituições passaram a cada vez mais a agir mais intensamente, com a ajuda da população, com finalidade de alcançar um produto com melhorias na própria qualidade, incluindo o alimento. (GOMES, 2004)

As cooperativas possuem o objetivo de possibilitar benefícios econômicos a seus membros; desenvolver a habilidade econômica e financeira dos membros unido ao mercado; diminuir o gasto de obtenção de produtos de consumo dos colaboradores e autoriza a função de produtos ou empregos no mercado por custos mais concorrentes. Entretanto, autoriza um excelente controle de qualidade diante de produtos obtidos ao mercado de forma mais eficaz. Diante disso a sociedade cooperativa encontra-se como intermediária entre os agregados e suas afinidades com o mercado (POLONIO, 2004).

Uns dos fatores que vem contribuindo para o desenvolvimento social e também equilibrar o país é a agricultura familiar, visto que através de milhares 
de pequenos produtores é um campo de crescimento de grande importância para o nosso país. Sendo assim, tem participação relevante de criação de empregos, criando e distribuindo renda e consequentemente menos evasão do campo para cidades. (DAMASCENO; KHAN; LIMA, 2011),

De acordo com as pesquisa da FAO (Food and Agriculture Organization) e do INCRA (Instituto Nacional de Colonização e Reforma Agrária), os órgãos governamentais aos poucos vem provendo a entrada democrática voltada aos recursos produtivos, fazendo com que diminui as desigualdades e aperfeiçoando o bem estar dessas famílias, através do recurso de implementação de mecanismo de incrementar a produtividade, e ao mesmo tempo combatendo a fome e geração de novos empregos e renda (DELGADO; BERGAMASCO, 2017).

A grande parte das DTA’s (Doenças Transmitidas por Alimentos) se prolifera por uma série de erros no processo produtivo, como por exemplo, falha na manipulação, temperatura inadequada, má conservação dos alimentos, deficiência na higiene pessoal, manipulador contaminado juntamente com o alimento já pronto e entre outros. As Boas Práticas de Manipulação (BPM) de alimentos garante o cuidado do alimento que futuramente será consumido por pessoas estando livre de contaminação (SOARES, 2006).

Os procedimentos de Boas Práticas de Fabricação abordam medidas a serem tomadas por indústrias alimentícias e pequenas propriedades, garantindo um alimento seguro para o consumo. Deste modo, existem legislações que regulamentam medidas técnicas para garantir a inocuidade dos alimentos. Os alimentos devem está livre de contaminação, ou seja, que não haja presença de agentes químicos, biológicos e físicos que possam acarretar contaminação. Todos os colaboradores (funcionários) que tenham contato com alimentos devem receber, no seu cotidiano de trabalho, as instruções necessárias e atividades a serem cumprida para garantir um alimento seguro e de qualidade ao final de sua preparação (WERNER, 2018).

O sistema de qualidade e a preservação da higiene são essenciais à implantação, pois tem uma importância quando manipula os alimentos. No entanto, nas maiorias das vezes, é descuidado, ou seja, feita em condições inapropriadas, fazendo assim que o microrganismo se desenvolva contaminando o alimento (SOARES, 2006).

Boas práticas são preceito com métodos de atingir um padrão determinado de garantir a qualidade do produto e serviço, quando colocada em prática 
auxilia na prevenção de certos perigos. Dessa forma, abrange Higiene dos utensílios, equipamentos e ambiente, garantia de qualidade da água, controle de praga, higiene pessoal adequada e entre outros temas (SILVA, 2001). Mediante a isso, os resultados coletados através da aplicação do Check list de BPF, verificação da adaptação dos funcionários às normas de BPF, possibilitar em delinear as possíveis medidas para a contemplação da cooperativa em relação a legislação vigente. Caso o estabelecimento de manipulação não apresentar um controle, pode haver um desencadeamento de uma possíveis doenças transmissíveis por alimentos (DTA), para evitar ou minimizar necessita-se adotar as regras em relação às Boas Prática de Manipulação, e assim fornecendo uma alimentação segura aos consumidores

Diante das questões envolvidas em relação à qualidade dos alimentos, as Boas Práticas de Fabricação permitem um ambiente de trabalho mais eficaz, contribuindo para a eficiência de um processo de produtivo. São relevantes para controlar certas fontes de contaminação cruzada e sendo assim para garantir que o produto final atenda as necessidades de especificações da identidade e de qualidade (MACHADO; DUTRA; PINTO, 2015).

Com a grande demanda do mercado da alimentação, torna essencial ter um diferencial nesse meio competitivo nas empresas desse ramo da alimentação tendo a qualidade dos produtos mais agregada, fazendo que através desse diferencial determine quais empresas vão se destacar e posteriormente permanecerão. Umas das vantagens competitivas é a qualidade que diferencia uma empresa de outra, pois cada vez mais os consumidores estão cientes e exigentes a qualidade dos seus produtos de onde vieram. $\mathrm{E}$ as empresas que não optar por essa preocupação que é o diferencial no mercado que poderá ficar de fora dentre as melhores (TRÄSEL, 2014).

Além da grande troca de conhecimento entre estudante executante do projeto e os manipuladores de alimentos, o trabalho consegue promover a produção de um alimento íntegro, seguro, e de melhor qualidade para a comunidade em geral. De acordo Artur (2004), os alimentos são considerados íntegros e seguros quando estão próprios para o consumo humano atendendo aos critérios de não causar infecção ou intoxicação alimentar quando manipulados e preparados de maneira adequada.

Diante desse fato o presente estudo teve como objetivo o acompanhamento de Boas Práticas de Manipulação em uma cooperativa agroindustrial do município de Naviraí/MS, avaliando a qualidade dos produtos elaborados por 
meio de um questionário, Esta legislação, de abrangência nacional, estabelece os procedimentos a serem adotados para garantir a qualidade e a segurança dos alimentos manipulados.

\section{METODOLOGIA}

A Cooperativa pesquisada está localizada na área rural do município de Naviraí-MS, fabrica pães e possui um frigorífico de frangos. Os funcionários são as famílias que moram nessa área rural, que contam com 25 pessoas, com faixa etária entre 30 e 50 anos. Vinte cooperados foram submetidos à avaliação sobre conhecimentos básicos acerca das Boas Práticas de Manipulação de Alimentos. O Quadro 1 apresenta o questionário aplicado aos manipuladores para identificar o nível de conhecimento dos mesmos sobre as Boas Práticas de Manipulação de Alimentos.

QUADRO 1 - QUESTIONÁRIO APLICADO AOS MANIPULADORES DE ALIMENTOS DA COOPERATIVA.

\begin{tabular}{|c|c|}
\hline QUESTÃO & DESCRIÇÕES DAS PERGUNTAS \\
\hline 1 & $\begin{array}{c}\text { Assinale os itens que estão incluídos nas Boas Práticas de } \\
\text { Manipulação. }\end{array}$ \\
\hline 2 & $\begin{array}{c}\text { Com que frequência deve ser higienizada as instalações, os } \\
\text { equipamentos, móveis e utensílios? }\end{array}$ \\
\hline 3 & $\begin{array}{l}\text { Quais são os hábitos que precisam ser evitados durante a } \\
\text { manipulação para proteger os alimentos de contaminação? }\end{array}$ \\
\hline 4 & $\begin{array}{l}\text { Assinale o (s) fator (es) que podem provocar doenças de origem } \\
\text { alimentar. }\end{array}$ \\
\hline 5 & $\begin{array}{c}\text { Se o cloro acabar no seu local de trabalho, qual o outro meio de } \\
\text { higienização de utensílios e equipamentos que você poderia } \\
\text { utilizar? }\end{array}$ \\
\hline 6 & $\begin{array}{l}\text { Qual a diferença de água tratada e água potável? Quais das duas } \\
\text { podem ser utilizadas na manipulação de alimentos? }\end{array}$ \\
\hline 7 & $\begin{array}{c}\text { Como devem ser rotulados os alimentos preparados ou } \\
\text { fracionados, no local de comercialização e embalados na } \\
\text { presença do consumidor? }\end{array}$ \\
\hline 8 & Assinale qual faixa de risco para crescimento de microrganismos. \\
\hline 9 & $\begin{array}{l}\text { Quais são os cuidados que se deve ter no armazenamento para } \\
\text { não comprometer a qualidade e a segurança do alimento? }\end{array}$ \\
\hline
\end{tabular}

Para avaliação das condições higiênico-sanitárias dos estabelecimentos, utilizou-se um check-list elaborado com base legal, na Resolução RDC nº 275 
de 21 de outubro de 2002 (BRASIL, 2002) e na Resolução RDC n 216 de 15 de setembro de 2004 (BRASIL, 2004). Foram avaliadas as condições das instalações, edificações, equipamentos, móveis e utensílios a fim de verificar cada item listado. As condições higiênico-sanitárias e o modo operacional de sanitização foram analisados visualmente, e em seguida, percebeu-se a não existência de registros ou quaisquer documentos que provassem a forma e o local em que as mesmas foram feitas. A estrutura interna e externa do estabelecimento foi aferida para visualização do controle de pragas e vetores, sendo este item listado significativo para as futuras ações corretivas. Avaliou-se as condições higiênicas dos reservatórios de água, bem como tubulações e torneiras.

Para encerramento das atividades do projeto, foi ministrada uma palestra intitulada "Boas Práticas de Manipulação de Alimentos" para todos os manipuladores de alimentos dos estabelecimentos que participaram das atividades. Abordou-se a importância das Boas Práticas de Manipulação de Fabricação de Alimentos para a garantia da comercialização e consumo de um alimento seguro.

\section{RESULTADOS E DISCUSSÃO}

Foram observados que alguns pontos onde são elaborados os alimentos apresentavam falhas inclusive na sua própria elaboração. O Quadro 2 apresenta o nível de conhecimento dos manipuladores sobre as Boas Práticas de Manipulação de Alimentos.

QUADRO 2 - PORCENTAGEM DE ACERTOS E ERROS, APÓS A APLICAÇÃO DO QUESTIONÁRIO A FIM DE AVALIAR O CONHECIMENTO DOS COOPERADOS.

\begin{tabular}{|c|c|c|}
\hline Assunto Abordado & Porcentacem de ACERTOS (\%) & PorCentaGem De erros (\%) \\
\hline Hábitos higiênicos & $65 \%$ & $35 \%$ \\
\hline Segurança de alimentos & $55 \%$ & $45 \%$ \\
\hline Contaminação alimentar & $50 \%$ & $50 \%$ \\
\hline $\begin{array}{c}\text { Higienização dos } \\
\text { utensílios e equipamentos }\end{array}$ & $45 \%$ & $40 \%$ \\
\hline $\begin{array}{c}\text { Higienização do local de } \\
\text { manipulação }\end{array}$ & $60 \%$ & \\
\hline
\end{tabular}


Os resultados obtidos do questionário elaborado e respondidos pelos cooperados apresentou um resultado médio em algumas áreas, pois os mesmos não apresentam um grau de escolaridade básico ou completo. Os manipuladores de alimentos devem conhecer as normas sobre as condições higiênicas sanitárias e higiene pessoas. A ocorrência de uma possível enfermidade entre o manipulador e os alimentos, mesmo deve ser impedido a entrada para a área de manipulação de alimentos. Deste modo, os profissional envolvido deve passar por treinamento e exame de forma periódicas (BRASIL, 1997).

O check-list aplicado era composto por 50 itens divididos em 22 blocos. Os índices de conformidades então apresentados no Quadro 2.

QuAdro 2-PoRCENTAGEM DE CONFORMIDADES OBTIDA ATRAVÉS DO CHECK-LIST.

\begin{tabular}{|c|c|c|}
\hline $\begin{array}{l}\text { ITENS AVALIADOS/ } \\
\text { PORCENTAGEM DE } \\
\text { CONFORMIDADES }\end{array}$ & $\begin{array}{c}\text { AVALIAÇÃO DO FRIGORIFICO DE } \\
\text { AVES }\end{array}$ & $\begin{array}{c}\text { AVALIAÇÃO DO LOCAL DE } \\
\text { MANIPULAÇÃO DE PANIFICAÇÃO }\end{array}$ \\
\hline $\begin{array}{l}\text { Edificação, instalações, } \\
\text { equipamentos }\end{array}$ & $65 \%$ & $60 \%$ \\
\hline $\begin{array}{l}\text { Higienização de } \\
\text { instalações }\end{array}$ & $70 \%$ & $60 \%$ \\
\hline $\begin{array}{l}\text { Controle integrado de } \\
\text { vetores e pragas }\end{array}$ & $80 \%$ & $55 \%$ \\
\hline Abastecimento de água & $50 \%$ & $70 \%$ \\
\hline $\begin{array}{l}\text { Matérias-primas, } \\
\text { ingredientes e } \\
\text { embalagens }\end{array}$ & $50 \%$ & $90 \%$ \\
\hline Preparação do alimento & $95 \%$ & $60 \%$ \\
\hline $\begin{array}{l}\text { Higienização do local de } \\
\text { manipulação }\end{array}$ & $74 \%$ & $55 \%$ \\
\hline Atestados de saúde & $92 \%$ & $85 \%$ \\
\hline Armazenamento & $75 \%$ & $65 \%$ \\
\hline
\end{tabular}

Deste modo pode-se visualizar que o frigorífico de aves apresentou maior conformidade comparada com o setor de panificação. Em ambos os setores avaliados apresentou-se não conformidades tais como: Edificação, instalações, equipamentos, móveis e utensílios apresentaram falhas nas janelas, pia, rachadura nos pisos. A higienização do local de manipulação não é periódica.

O abastecimento de água apresentou falhas no modo que é usado, a mesma água da higienização do ambiente também era usada para lavar sujeiras 
grossas das mãos diretamente no tanque como, por exemplo, o sangue do abate dos frangos e foi necessária a mudança desses hábitos que poderia causar contaminação cruzada.

O setor de alimentação, em relação ao frigorífico de aves, apresentaram conformidade em relação aos itens abordados em relação aos relatórios técnicos. Podemos salientar que a carne é um grande meio de contaminação de uma possível DTA.

Já no setor de panificação não apresentava condições higiene sanitária de acordo com as legislações e resoluções vigentes em relação às boas práticas. Mediante a isso a área de panificação demonstrou não conformidade em relação à higienização dos equipamentos e maquinário e utensílios não são adequados, deve ser feito periodicamente e usando os produtos de limpezas adequados. Boas práticas do manuseio de lixos obteve-se uma aprendizagem eficaz no modo que era feito antes do presente projeto. Armazenamento e distribuição dos alimentos com o auxílio da palestra os manipuladores mudaram os hábitos errados de organização e com isso os seus afazeres ficaram mais práticos e rápidos no dia a dia.

Aplicação de uma cartilha sobre boas práticas de alimentos foi elaborada especialmente em cima das dificuldades dos cooperados, em uma linguagem de fácil entendimento.

A execução desse projeto trouxe benefícios significativos aos estabelecimentos e à comunidade, visto que melhorando a higiene na manipulação dos alimentos, garante-se que os produtos alimentícios comercializados nas padarias sejam seguros e de qualidade a população local.

No encerramento do projeto, foi ministrada uma palestra a todos os manipuladores de alimentos dos estabelecimentos participantes (Figura 1). A palestra foi ministrada por sete alunos do curso de Engenharia de Alimentos de Naviraí, todos participantes desse projeto de extensão, tendo como tema "Boas Práticas de Manipulação de Alimentos", onde foram abordadas informações básicas, desde a forma como se deve limpar uma caixa d'água, até à importância das Boas Práticas de Manipulação de Fabricação de Alimentos para a garantia da comercialização e consumo de um alimento seguro. Além da apresentação oral, ocorreram dinâmicas com a finalidade de envolver os manipuladores presentes, proporcionando trocas de conhecimentos..

Figura 1. Foto da Realização da Palestra sobre as Boas Práticas de Mani pulação 


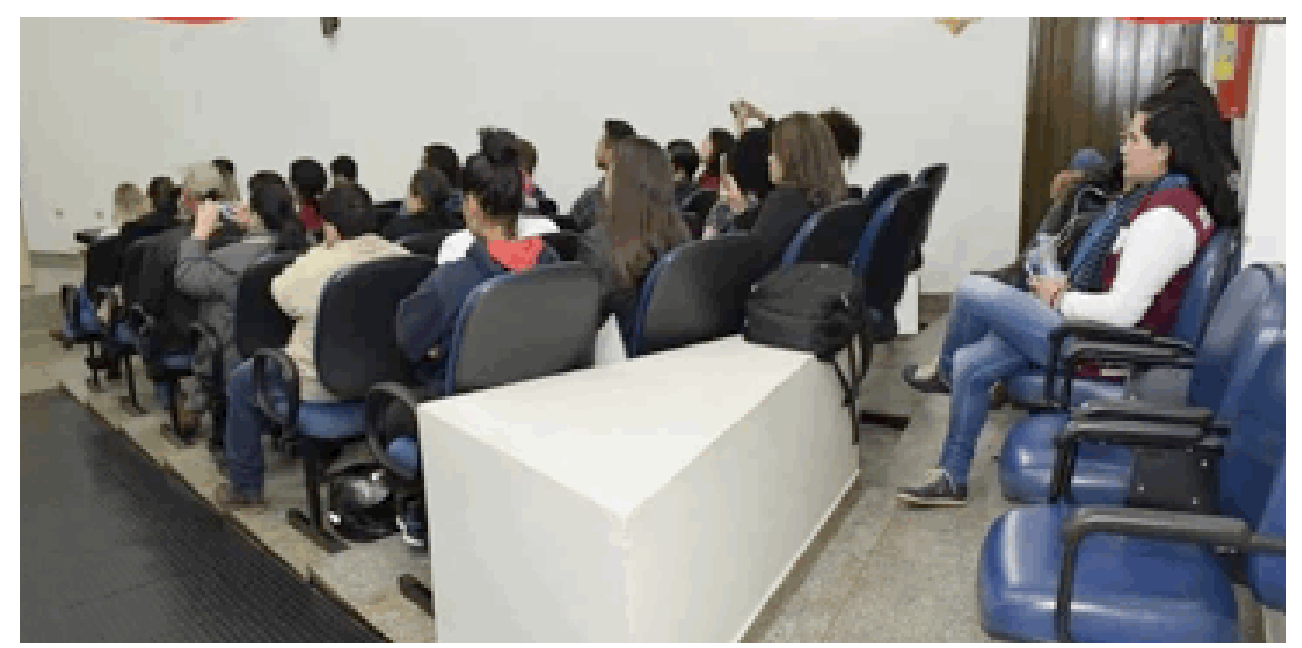

\section{CONSIDERAÇÕES FINAIS}

Alimentos manipulados de forma correta e segura são requisitos básicos para saúde dos consumidores. Desse modo pode-se salientar que a realização do projeto foi de extrema importância para ambas as partes, podendo assim, proporcionar uma troca de conhecimentos entre os acadêmicos e a instituição participante. Com o desenvolvimento do projeto, obteve-se boa aceitação e interação dos manipuladores de alimentos da cooperativa nas atividades, os quais solicitavam informações e opinavam sobre as possíveis melhorias no processo, com a finalidade de melhorar as condições higiênicas- sanitárias da fabricação, manipulação e qualidade dos alimentos. É notório que as ações corretivas difundiram conhecimento técnico, sobre a qualidade e segurança dos produtos acabados.

Ações corretivas como treinamentos e palestras serão realizadas e ajudarão os manipuladores de alimentos a garantir a qualidade dos alimentos produzidos. A criação e o desenvolvimento desse projeto de extensão vêm comprovando que, além de promover a interação entre os alunos e manipuladores de alimentos, cria-se um vínculo entre a universidade e a comunidade, fortalecendo a importância da atuação universitária no meio onde está inserida, não só como geradora de conhecimento, mas também de protagonismo social. A promoção do conhecimento adquirido tecnicamente na universidade pode ser usada beneficamente a favor da comunidade, de modo que haja essa promoção do relacionamento entre diferentes áreas de conhecimento a favor do bem comum. 


\section{REFERÊNCIAS}

ARTUR, P. de O. Aplicação do binômio tempo/temperatura em alimentos. 2004. 57 f. Trabalho de Conclusão de Curso (Especialização)-Universidade de Brasília, Brasília, 2004.

BIALOSKORSKI NETO, S. A nova geração de cooperativas e a coordenação dos sistemas agroindustriais. In: WORKSHOP Brasileiro de Sistemas Agroalimentares, 2., 2000, Ribeirão Preto. Acesso em: 07 maio 2018.

Brasil: estudo transversal de base populacional. Revista Panamericana de Salud Pública/ Pan American jornal of Public Health, Washington, v.16, n.5, p. 308-314, 2002. Acesso em: 06 maio. 2018.

BRASIL. MINISTÉRIO DA SAðDE - MS AGêNCIA NACIONAL DE VIGIL NCIA SANITÁRIA - ANVISA. (Org.). RESOLUÇÃO DE DIRETORIA COLEGIADA - RDC No 275, DE 21 DE OUTUBRO DE 2002. 2002. 23 p. Disponível em: http://portal.anvisa.gov. br/documents/10181/2718376/RDC_275_2002_COMP.pdf/fce9dac0-ae57-4de2-8cf9e286a383f254. Acesso em: 14 maio 2018.

BRASIL. Ministério da Saúde, Resolução Agência de Vigilância Sanitária. Resolução no 216, de 15 de setembro de 2004. In: DIÁRIO OFICIAL DA UNIÃO. Brasília, 16 de setembro de 2004. Acesso em: 06 maio. 2018.

BRASIL, Ministério da Saúde. Agência Nacional de Vigilância Sanitária. Portaria n 326, de 30 de julho de 1997. Regulamento técnico sobre as condições higiênico-sanitárias e de boas práticas de fabricação para estabelecimentos produtores/industrializadores de alimentos. Brasília, Diário Oficial da União, 1 de ago. 1997.

DAMASCENO, Nagilane Parente; KHAN, Ahmad Saeed; LIMA, Patrícia Verônica Pinheiro Sales. O Impacto do Pronaf sobre a Sustentabilidade da Agricultura Familiar,Geração de Emprego de Renda no Estado do Ceará.RESR, Piracicaba, SP, vol. 49, nº 01, p. 129-156, jan/mar 2011- impressa em maio 2011. Acesso em: 07 maio 2018

DElGado, Guilherme Costa; BergamasCO, Sonia Maria Pessoa Pereira. Agricultura Familiar Brasileira: Desafios e Perspectivas de Futuro: Secretaria Especial de Agricultura e do Desenvolvimento Agrário. Brasília: 2017. Disponível em: file://C:/Users/ UEMS/Documents/1-\%20Agricultura\%20Familiar\%20Brasileira\%20Desafios\%20e\%20 Perspectivas\%20do\%20Futuro.pdf. Acesso em: 09 maio 2018.

FLORES, S. A. M; GREGORI, R; SILVEIRA, G. E. Análise da estrutura das cooperativas agroindustriais do Rio Grande do Sul, 2010. Disponível em www.abepro.org.br/biblioteca/enegep2010_tn_st o_115_753_15569.pdf. Acesso em: 06 maio. 2018.

GOMES, Patrícia Machado. Avaliação do processo de implantação de boas práticas de manipulação de restaurantes de Brasília-DF. Brasília: Gstdghtsdj, 2004. Disponível em: http://bdm.unb.br/bitstream/10483/558/1/2004_PatriciaMachadoGomes.pdf. Acesso em: 14 maio 2018. 
LIMA, Ozeir Celestino de; SILVA, Wilkcimara Santiago. Agricultura Familiar: análise a partir da fundamentação de autores a cerca do Programa Nacional de Fortalecimento da Agricultura Familiar - PRONAF. Disponível em: http://www2.ufersa.edu.br/portal/ view/uploads/setores/241/Agricultura\%20Familiar\%20-\%20Enviar.pdf. Acesso em: 07 maio 2018.

MACHADO, Roberto Luiz Pires; DUTRA, André de Souza; PINTO, Mauro Sergio Vianello. Boas Práticas de Fabricação (BPF). Rio de Janeiro: Embrapa, 2015. Disponível em: https://ainfo.cnptia.embrapa.br/digital/bitstream/item/132846/1/DOC-120.pdf. Acesso em: 09 maio 2018.

POLONIO, W. A. Manual das sociedades cooperativas. 4. ed. São Paulo: Atlas, 2004.

RIO, A. P. D. Manual de boas práticas de fabricação em indústria de gelo, 2004.

Disponível em: http://bdm.unb.br/bitstream/10483/206/1/2004_AlessandroPereiraDelRio.pdf\&gt. Acesso em: 09 maio 2018.

SILVA JUNIOR, E. A. da. Manual de controle higiênico-sanitário em alimentos. São Paulo: Varela, 2001.

SOARES, Antônio Gomes (Org.). Boas Práticas de Manipulação em Bancos de Alimentos. Rio de Janeiro: Embrapa, 2006. 31 p. Disponível em: https://ainfo.cnptia.embrapa. br/digital/bitstream/item/169320/1/Doc-74-Boas-Praticas-de-Manipulacao-2006.pdf. Acesso em: 07 maio 2018.

TRÄSEL, Karoline. IMPLANTAÇÃO DE BOAS PRÁTICAS DE FABRICAÇÃO EM EMPRESA DE CHOCOLATES ARTESANAIS EM ARROIO DO MEIO - RS. Lajeado, 2014. Disponível em: <https://www.univates.br/tecnicos/media/artigos/Karoline.pdf >. Acesso em: 07 maio 2018.

VELÁSQUEZ-MELÉNDEZ, G.; PIMENTA, A. M.; KAC, C. Epidemiologia do sobrepeso e da obesidade e seus fatores determinantes em Belo Horizonte (MG). Rev. Panam. Salud Publica, 2004, 16 (5). Disponível em: https://www.scielosp.org/pdf/rpsp/2004. v16n5/308-314/pt. Acesso em: 07 maio 2018.

WERNER, T. Manual de boas práticas. Disponível em: http://www.ticianawerner.com.br/ docs/manualboaspraticas_ticianawerner.pdf. Acesso em: 09 maio 2018. 